\title{
ETIMOLOGIE VENETE
}

Non è per emulare un grande studioso, come Angelico Prati, che richiamiamo il titolo di una pregovole raccolta (Etimologie venete, Venezia-Roma, 1968), ma soltanto per contribuire alla sua integrazione con l'analisi di alcune voci in essa non comprese.

bajàn (pad.) "contadino, villano" (anche bajanóto). - Dal significato più generico di "babbeo" (baggiano), che bagiàn ha in diversi dialetti veneti (venez., chiogg.) e trentini (compreso l'anaunico-solandro).

bassabréve (pad.) "plebaglia, popolino, gente povera". - Travisamento del sintagma dotto bassa plebe.

bonamàn (venez., pad., rovig., vic., ver., trev., ampezzano, bisiaco, cembrano, anaunico-solandro, friul.) "mancia, regalo", specialmente (venez., trev., feltr., agord., trent., friul.) o solamente (valsug., zoldano, ven. giul., ven. dalm., fiumano) "strenna di Capodanno o Natale" (cfr. AIS IV $781 \mathrm{Cp}$.). La formula, di probabile provenienza dai dialetti settentrionali, è ampiamente diffusa, nelle due versioni bonamàn e bambóna, anche nei paesi ladini (J. Kramer con la collaborazione di S. Kowallik in "Mondo ladino" X, 1986 = Studi ladini in onore di Luigi Heilmann, pp. 316-617). - La spiegazione tradizionale (da bona man "mano generosa" e, poi, "mancia, dono di capodanno") è stata posta in dubbio da R. Zeli nel Voc. dial. Svizzera ital. s.v. bonamàn, e sostituita da un più convincente bona mane "buon mattino", riferito all'augurio fatto alla mattina presto del primo giorno dell'anno, durante la questua, come confermerebbe il testo della filastrocca, che l'accompagna.

capèa (pad.) "cappella“ nella loc. essare o stare soto 'la capèa de qualcheduno "essergli sottomesso". - Dal gergo dei muratori, per i quali cappella è il "gruppo di muratori dipendenti dal medesimo capomastro", secondo la definizione di A. Menarini, I gerghi bolognesi, Modena,, 1942, dove, s.v. kapèla, è detto: "Ancor oggi usato, senza intenzioni gergali. Es.: a san satta la kapèla ed Martèl = Sono alle dipendenze di Martelli. A Porretta muratori e scalpellini chiamano ora ed cappella l'ora di colazione". Significati affini ha il francese, dove chapelle, dal primitivo significato di "insieme di ecclesiastici, che servono in una cappella", è passata nell'Ottocento a designare tanto una "associazione di mutuo soccorso tra operai“, quanto (nell'argot) la "riunione di operai, che discutono dei loro interessi corporativi“. 
capeàn (pad.), capelàn (trev., vic., valsug., cembrano) "cappelano" nella loc. andare da (o par) cape(l)àn "detto del marito, che va a vivere con i suoceri". - Dalla posizione di dipendenza del cappellano dal parroco. La locuzione s'incontra nel Trevisano e nella Valsugana con il tipo equivalente "andare cucco", che prevale a nord, mentre verso est è dominante il tipo veronese nare a grumbiale: il primo allude all'abitudine del cuculo di deporre le uova nel nido altrui, l'altro al grembiule, come indumento proprio delle donne. V. anche "Veneto ieri, oggi, domani" I 9 (settembre 1990), p. 120.

de baruccabà (pad.) "con mezzi traversi", "di provenienza dubbia, generalmente compendio di furto". - Sembra dipendere, attraverso una semplice simpatia fonosimbolica, dall'ebraico baruhabà, letteralmente "benedetto colui che viene", espressione augurale di benvenuto, ed anche "salmo nuziale", ma altresi nome del protagonista di alcune canzoni antisemitiche (da cui furono tratte pure fortunate commedie) del XVIII secolo, variamente chiamate Storia di Baruccabà, Il secondo sposalizio di Baruccabà, Diana infedele di Baruccabà, ecc. Della loro popolarità fa fede il detto buranese la n'a fatto [pì] de barucapà vinturina, ló nà fatto pi de Baruccabà él venturièr "ne ha fatto più di Bertoldo". Da staccarsi da questa tradizione, per ragioni cronologiche, è l'aretino baruccabà "confusione“, che il Caix (Studi di etim. it. e romanza, Firenze, 1878, p. 76) trae dal Redi e spiega con la frequenza di bārūch habbāh nelle preghiere degli Ebrei (cfr. J. Babad in Z.R.Ph. XVII, 1893, pp. 562-563).

maramàn (chiogg.) "maramaldo, meridionale", (pad.) "persona inavvicinabile, trasandata", (a Montagnana) "persona poco socievole e trasandata nell'abbigliamento; rozzo", (ver.) "turbolento, selvaggio, inavvicinabile", (a Garda, al pl.) "ragazzi scapestrati e violenti", (cembrano) "marame, accozzaglia", (alto trev.) "poco di buono", (friul.) "terrone, meridionale". - Da maremmano: "Siccome i Maremmani sono generalmente di aspetto e di modi un poco rozzi; così suol dirsi: Pare un Maremmano, a chi è rozzo nel vestire e nel procedere" (TommaseoBellini Giunte), come nota anche Romano Bilenchi in un racconto (Un errore geografico) compreso nella raccolta Mio cugino Andrea: "da noi maremmano è sinonimo d'uomo rozzo e ignorante".

mòma (pad.), secondo il Patriarchi, è la "spia", specie nella loc. far la moma "far la marachella, raccoglier spinacci", che possiamo intendere, sulla base dell'uso contemporaneo, "osservare e seguire di soppiatto i movimenti di qualcuno: il gatto, per esempio, che fa la posta al topo, el ghe fa 'la mòma. - Fa parte di una serie di modi espressivi, come girare 'la mòmo'la "gironzolare senza meta, perder tempo“, ndare de momo'lón "aggirarsi in un luogo, talvolta con fare sornione“, momo'lare, che, oltre al significato di (valsug., rover.) "biascicare", ha nel pad. quello di "cincischiare, perdere tempo, non concludere nulla"; così (pad.) momo'lón è il "girondolone", il "perditempo".

mòmolo (ver.) "cucirino di cotone da imbastire". - Voce isolata. L'unico accostamento è al furbesco venez. sior Momolo "membro virile" (per metafora). 
ómbro (pad. sett.) "la conta nei giochi infantili“: "In questo gioco [dei bottoni] incideva molto la fortuna d'essere sorteggiati per primi, e questo si faceva con l'ombro che era una specie di tòcco, o di cònta“ (U. Azzalin, Usanze costumi e mangiari di paese, Vicenza, 1973, p. 42). - Dal lat. numerus, come (ver., trent.) ombrar è da numerare attraverso (rov.) nombrare e (vic., valsug.) lombràr(e) (Prati EV), l'uno e l'altro bene attestati in pavano.

pàcara (pad., vic.) "scavatrice meccanica“, pàchera (trent., anaunico-solandro) "id."; anche in friul. pàcare. - Dal ted. Bagger "id.", secondo la pronuncia tirolese, come hanno riconosciuto autori di vocabolari dialettali (Quaresima, Scalfi) e G. Riedmann, che testimonia la presenza di pachera anche nella stampa altoatesina in italiano ("Alto Adige") dal 1966 (Die Besonderheiten der deutschen Schriftsprache in Südtirol, Mannheim-Wien-Zürich, 1972, p. 38).

peapà nella loc. (venez.) lexer el peapà "imparare il padre nostro", beabà (trev., a Vittorio Veneto; ampezzano), biabà (trent.) "sillabario". - Dal procedimento didattico d'insegnare a compitare, partendo dalla lettura delle lettere ( $p e, b e, a) \mathrm{e}$ unendole in sillabe $(p a, b a)$. Anche in catalano beabà "sillabario".

perlustrato (pad. a Ospedaletto) "giovane male in arnese, disordinato nel vestire, trasandato in ogni cosa, con capelli e barba incolti, senza arte né parte, lazzarone“, anche nelle varianti pe'lustrato, pi'lustrato (nel quartiere urbano del Portello) "persona vestita male, scalcinata“, peustrà (a Boion, tra Padova e Venezia) "soldato di leva" (ma questo si può confrontare con l'ampezzano peluštro "ronzino", cioè 'pelo lustro'; si tratta, quindi, probabilmente, di sovrapposizione di un significato diverso, dopo la perdita del valore proprio della voce), noto anche nel Vicentino (L. Meneghello in Libera nos a malo ricorda balustrato) e nel Veronese (perlustrato "scansafatiche"). - Da perlustrato o balustrato, che il Mazzucchi definisce: "Perquisito (nel senso d'individuo condannato dall'Austria al forzato servizio militare)“, noto anche al Friuli (pilustrât) assieme al verbo pilustrâ. Di qui la locuzione ver. nare perlustrato "partire per un lustro: (leva militare austriaca che durava, appunto, cinque anni)“ (D. Coltro, Paese perduto. La cultura dei contadini veneti, III: Le parole del moléta, Verona, 1975, p. 321: si tratta di una interpretazione paretimologica), spiegata con la frequente frase: Caro, 'na 'olta $i$ preti $i$ te podea far 'ndar via pelustrato! ( = al confino di polizia), citata da G. Manzini in AA.VV., Il mondo agrario-tradizionale nella valle padana. Atti del Convegno di studi sul folklore padano, Modena, 1963, p. 214. Altre notizie nella rivista "Veneto ieri, oggi, domani“ I 5 (maggio 1990), p.120.

po'lara (pad., a S. Gregorio) "pioppo" (a Brugine polara è il "tremolo, Populus tremula": ALI), nome che conferma la variante (assimilata o erronea) accolta dal Penzig proprio da Padova palaro "Populus alba L.“. - Dal lat. (arbor) *popularia "albero del pioppo", presente, forse, anche nel parm. piopa pirara "pioppo cipressino, pioppo piramidale. Specie di pioppo che s'alza a modo del pino e del cipresso. Il Populus fastigiata di Linn“. L'agg. *popularius si è conservato sia nel fr. peu- 
plier (ma per il FEW IX 182 si tratta dell'aggiunta a populus del suff. -ier proprio degli alberi da frutta), sia nella toponomastica ven. e friul.: Paularo, Povolaro (Pellegrini-Zamboni).

prefàssio "prefazio, parte della messa, con la quale hanno inizio il canone romano e le altre preghiere eucaristiche“, vivo, soprattutto, nella loc. (venez.) ochio [ocio] del prefazio "cipiglio, guardatura torva", (rov.) guardare con l'òcio del prefàzio "guardare in cagnesco", (ver.) vardàr con l'òcio del prefásio "vedere di mal occhio, stare in sospetto di qualcuno", (v)ardàr co' l'ocio del prefassio "di sottecchi“, (trent.) vardar coi òci del prefazio "guardare con sospetto, con meraviglia“. La locuzione è anche del lucchese: guardar coll'occhio del Prefazio "in tralice; storto, torvo". - Il modo, frutto di attenta osservazione, si rifà all'atteggiamento del celebrante, che, fermo al centro dell'altare, deve leggere il messale posto al suo fianco, inclinando lo sguardo.

fòbri (pad.) nel gioco del mondo (campanón o sca'lón) "fallo!", che si ha quando il giocatore cerca di percorrere, ad occhi chiusi, tutte le caselle, dicendo: $A n$ ?, cui il compagno di gioco risponde: - Salan!, se il piede entra nella casella, oppure: - Jòbri, se pesta una delle linee, che le divide. - Metatesi, accidente non raro nel gergo padovano, di bruso ( ( òbru con la rara vocale di uscita modificata in -i). Bruso, come nome (di questa fase) del gioco, è attestato in vicentino (Val Leogra) e in Friuli (V. Ostermann Arch. tradiz. pop. IX, 1890, p. 567) e la domanda: - Brucio?, al posto di $-A n$ ?, è usata tanto nel Modenese (Neri ss.vv. bru $\int a$ e settimana), quanto in area folignate-spoletina (R. Bruschi nei Contributi di dialettologia umbra III 4, 1985). Si veda, inoltre, il DESF s.v. bruse ${ }^{3}$.

stèfani (ven., pad., vic., ver., poles.) "denti“. - E' voce piuttosto gergale (stèfeni in tarom), suggerita da "una specie di personificazione". (Migliorini Dal nome proprio 240). Di simile estrazione sono l'antico stèfano "stomaco" e l'agordino stéfani "natiche", specie nella loc. muove i stéfani "camminare, andare di buon passo", ampezzano menà $i$ štèfane "sbrigarsi".

sunanza (venez., chiogg., pad.) "replezione di stomaco", (pad., anche sumansa, sumassa e sumaza) solo nella loc. fare na sumansa "fare indigestione". - Il significato originario è "raccolta", da sunàre (Prati EV), che il LEI I 872-873 colloca s.v. adūnāre, con la sostituzione del suffisso ad-con ex-.

\section{Povzetek}

\section{BENEŠKE ETIMOLOGIJE}

Ugotavljajo se do sedaj še neznane etimologije beneških narečnih besed: bajàn 'kmet'; bassabreve 'neuko ljudstvo'; bonamàn 'napitnina, darilo'; capèa 'kapela'; capeàn v pomenu 'žveti pri ženinih starših'; de barucabà 'živeti od goljufije'; maramàn 'odljuden človek'; mòma 'ogleduh'; ombro 'število pri otroških igrah'; pàcara 'bager'; peapà 'črkovati'; perlustrato 'razcapanec'; po'lara 'topol'; prefàssio '(gledati) postrani'; Sòbri 'zmota'; stèfani 'zobje'; sunanza 'prebavna motnja'. 